\title{
Impacto dos transtornos do sono sobre o funcionamento diário e a qualidade de vida'
}

\author{
Sleep disorders impact on daily functioning \\ and life quality
}

\author{
Mônica Rocha MÜLLER² \\ Suely Sales GUIMARÃES
}

\begin{abstract}
Resumo
Os distúrbios do sono provocam conseqüências adversas na vida das pessoas por diminuir seu funcionamento diário, aumentar a propensão a distúrbios psiquiátricos, déficits cognitivos, surgimento e agravamento de problemas de saúde, riscos de acidentes de tráfego, absenteísmo no trabalho, e por comprometer a qualidade de vida. Este estudo foi realizado com o objetivo de revisar a literatura especializada sobre as características dos distúrbios de sono mais freqüentes na população geral e suas implicações sobre os comportamentos, a rotina diária e a qualidade de vida das pessoas portadoras dessa condição. Os estudos revisados mostram que os distúrbios do sono desencadeiam conseqüências adversas à saúde e ao bem-estar dos indivíduos, afetando o trabalho, a cognição, os relacionamentos e o funcionamento diário, com diferentes desdobramentos a curto, médio e longo prazo.
\end{abstract}

Unitermos: atividades diárias; transtornos do sono; qualidade de vida.

\begin{abstract}
Sleep disorders are responsable for many adverse outcomes on people's lives by reducing their daily functioning, increasing psychiatric disorders susceptibility, cognitive deficits, onset and aggravation of health problems, traffic accident risks, work absenteeism, and by diminishing the quality of life. This study was aimed to review the specialized literature about the characteristics of the sleep disturbances that present greater incidence in the general population, and its implications on affected people's behaviors, daily routine and quality of life. Sleep disorders were found to trigger adverse outcomes to people's health and well being by disturbing their work, cognition, relationship and daily functioning, with multiple unfolding in short, medium and long time.
\end{abstract}

Uniterms: activities of daily living; sleep disorders; quality of life.

O sono é uma função biológica fundamental na consolidação da memória, na visão binocular, na termorregulação, na conservação e restauração da energia (Reimão, 1996), e restauração do metabolismo energético cerebral (Ferrara \& De Gennaro, 2001). Devido a essas importantes funções, as perturbações do sono

VVVV

1 Artigo elaborado a partir da dissertação de M.R. MÜLLER, intitulada "Implicações dos transtornos do sono nas atividades e na vida diária da pessoa acometida". Instituto de Psicologia, Universidade de Brasília. 2004. Este estudo contou com apoio financeiro da CAPES.

2 Universidade de Brasília, Instituto de Psicologia, Departamento de Psicologia Escolar e do Desenvolvimento. Campus Universitário Darcy Ribeiro, ICC-Sul, 70910-900, Asa Norte, Brasília, DF, Brasil. Correspondência para/Correspondence to: M.R. MÜLLER. E-mail: <muller@unb.br>. 
podem acarretar alterações significativas no funcionamento físico, ocupacional, cognitivo e social do indivíduo, além de comprometer substancialmente a qualidade de vida.

O sono normal varia ao longo do desenvolvimento humano quanto à duração, distribuição de estágios e ritmo circadiano (Poyares \& Tufik, 2002; Thorleifsdottir, Björnsson, Benediktsdottir, Gislason \& Kristbjarnarson, 2002). As variações na quantidade de sono são maiores durante a infância, decrescendo de 16 horas por dia, em média, nos primeiros dias de vida, para 14 horas ao final do primeiro mês e 12 horas no sexto mês de vida. Depois dessa idade o tempo de sono da criança diminui 30 minutos ao ano até os cinco anos. Na vida adulta decresce a quantidade e varia o ciclo do sono em função da idade e de fatores externos. Com o avanço da idade, ocorrem perdas na duração, manutenção (Ferrara \& De Gennaro, 2001) e qualidade (Tribl et al., 2002) do sono. A dor, o uso de medicações e diferentes condições clínicas são exemplos de fatores que podem afetar a quantidade e a qualidade do sono, especialmente entre idosos, que são mais propensos a essas condições (McCrae et al., 2003).

Além da qualidade de vida, os distúrbios do sono também comprometem a segurança pública, porque aumentam o número de acidentes industriais e de tráfego (Martinez, 1999). As estimativas sobre o índice de acidentes e mortes causados por sonolência ou cansaço variam de $2 \%$ a 41\%, com alto custo em termos financeiros e da própria vida (Ferrara \& De Gennaro, 2001).

Este estudo revisa os avanços da literatura especializada sobre o impacto dos distúrbios de sono na vida das pessoas, incluindo pesquisas empíricas, revisões e artigos teóricos e livros técnicos publicados entre os anos de 1998 e 2006, além de manuais de classificação nosológica. A busca da literatura foi realizada nos bancos eletrônicos Medline, Psycholnfo, SciELO e Science Direct, utilizando as seguintes palavras chaves em diferentes combinações: sleep disturbance, quality of life, sleep hygiene, sleep deprivation, outcomes, insomnia, sleep apnea, somnambulism, bruxism, restless legs syndrome, periodic limb movement. Foram incluídos nesta revisão os artigos que faziam, no abstract, menção às conseqüências ou implicações dos transtornos do sono em geral, ou dos transtornos específicos na população 520 maior de 18 anos. Este trabalho permitiu a descrição das características dos distúrbios de sono mais freqüentes na população geral e a sistematização de suas implicações sobre os comportamentos, a rotina diária e a qualidade de vida das pessoas acometidas.

Os numerosos distúrbios do sono são definidos na literatura em três principais sistemas de classificação:

1. Classificação Internacional dos Distúrbios do Sono (ICSD) (American Sleep Disorders Association, 1997): classificação detalhada e a mais utilizada pelos profissionais da área da medicina do sono.

2. Manual Diagnóstico e Estatístico de Transtornos Mentais - DSM-IV-TR (American Psychiatry Association, 1994): classificação psiquiátrica que divide os transtornos do sono em (a) primários; (b) relacionados a transtornos mentais; (c) relacionados à condição médica geral e (d) induzidos por substância.

3. Classificação Internacional de Doenças - CID10 (World Health Organization, 1997): classificação utilizada por médicos em geral, que agrupa os transtornos em (a) distúrbios do início e da manutenção do sono; (b) distúrbios do sono por sonolência excessiva ou hipersonia; (c) distúrbios do ciclo vigília-sono; (d) apnéia de sono, (e) narcolepsia e cataplexia; (f) outros distúrbios do sono e (g) distúrbios do sono não especificados.

Esses três sistemas classificatórios são independentes e utilizam diferentes critérios de inclusão, causando dificuldades epidemiológicas, de diagnóstico e de interpretação de resultados sobre os diferentes distúrbios do sono. Dentre essas definições, a da insônia é especialmente difícil (Buysse \& Ganguli, 2002; Harvey, 2001; Roberts, Roberts \& Chen, 2002).

Na maioria das vezes, a insônia é definida como a queixa de "dificuldade para iniciar ou manter o sono", "sono não reparador" (Littner et al., 2003; Novak, Shapiro, Mendelssohn \& Mucsi, 2006), "despertares noturnos" (Rocha et al., 2002b), ou conforme os critérios de escalas de freqüência ou intensidade desses sintomas (Ohayon \& Roth, 2001). A variação nos critérios diagnósticos, tanto em número quanto na duração dos sintomas e associação com outros distúrbios do sono e transtornos mentais, dificulta a comparação de resultados entre estudos (Ohayon \& Hong, 2002; Ohayon \& Shapiro, 2002; Ohayon \& Smirne, 2002; Rocha, Gerra \& Lima-Costa 2002a; Rocha et al., 2002b). 
O entendimento da insônia como sintoma ou como síndrome também dificulta a comparação entre estudos (Eddy \& Walbroehl, 1999; Ohayon \& Shapiro, 2002). Como sintoma, a insônia é secundária às condições médicas, psiquiátricas ou ambientais. E como síndrome, ela é uma desordem primária que requer tratamento direto.

Para Hauri (1998), a insônia pode ser classificada conforme sua duração em (a) transitória, durando alguns dias; (b) de curto período, durando algumas semanas e (c) crônica, persistindo por meses ou anos.

O Consenso Brasileiro de Insônia a conceitua como a dificuldade de iniciar e/ou manter o sono e sono não reparador, comprometendo as atividades diárias. Esse critério leva em conta a associação da insônia com transtornos psiquiátricos, distúrbios do sono, fatores ambientais, higiene do sono inadequada, doenças e uso de substâncias (Poyares \& Tufik, 2002).

As definições de apnéia obstrutiva do sono e de narcolepsia também apresentam inconsistências e peculiaridades conceituais. A apnéia é uma síndrome caracterizada por paradas respiratórias totais (apnéias) ou parciais (hipopnéias) recorrentes durante o sono (Giles et al., 2006; Gus, Nunes e Silva, Fernandes, Cunha \& Sant'Anna, 2002; Victor, 2004), ronco excessivo, e despertares que fragmentam o sono e causam hipersonolência diurna (Bounhoure, Galinier, Didier \& Leophonte, 2005; Brown, 2005). A apnéia pode causar enfermidades cardiovasculares como hipertensão arterial, acidente vascular cerebral e hipoxemia (McNicholas, 2003; White, 2006).

O índice de apnéia e de hipopnéia (IAH) que um indivíduo apresenta é calculado pela média do número de episódios ocorridos em uma hora de sono. No diagnóstico da apnéia, o IAH pode ser considerado com cinco ou dez episódios, acompanhados por hipersonolência (Silverberg, laina \& Oskenberg, 2002).

Para o diagnóstico de narcolepsia, Silber, Krahn e Olson (2002) consideram que uma das dificuldades é a análise da hipersonolência diurna, um dos principais sintomas, que também pode ser resultado de distúrbios respiratórios ou de sono insuficiente. Outra dificuldade é o diagnóstico diferencial entre cataplexia autonômica não específica e cataplexia comportamental diante de um estímulo emocional. Os autores propuseram um critério para o diagnóstico da narcolepsia, utilizando informações clínicas e neurofisiológicas para confirmar ou não a ocorrência de cataplexia, sonolência excessiva não decorrente de outros distúrbios e início precoce do sono REM.

\section{Classificação internacional dos distúrbios do sono}

A Classificação Internacional dos Distúrbios do Sono (ICSD) (American Sleep Disorders Association, 1997) é um dos sistemas classificatórios mais utilizados na literatura especializada, incluindo quatro grupos organizados em subgrupos: dissonias, parassonias, distúrbios do sono associados a alterações médicopsiquiátricas e distúr-bios do sono propostos.

\section{Dissonias}

Dissonias são transtornos primários relacionados à iniciação ou manutenção do sono ou à sonolência excessiva, com distúrbios na qualidade, quantidade ou regulação de ritmo do sono (American Psychiatry Association - APA, 1994). As dissonias formam o maior grupo dentre os transtornos do sono, com 34 distúrbios subdivididos em distúrbios intrínsecos, extrínsecos e relacionados ao ritmo circadiano:

1. Distúrbios intrínsecos do sono: incluem 13 transtornos de etiologia orgânica (Reimão, 1996), como insônias psicofisiológica e idiopática e síndrome de movimentos periódicos dos membros.

2. Distúrbios extrínsecos do sono: incluem 14 transtornos desencadeados por fatores externos ao organismo e capazes de alterar o sono (Reimão, 1996), como higiene do sono inadequada e insônia por alergia alimentar.

3. Distúrbios do sono relacionados ao ritmo circadiano: incluem sete transtornos (Reimão, 1996) associadas à hipersonolência diurna (APA, 1994), como a síndrome da mudança rápida de fuso horário (jet lag) e transtorno do sono por trabalhos em turnos.

\section{Parassonias}

Parassonias são alterações comportamentais ou fisiológicas que ocorrem em diferentes momentos do 
sono e formam o segundo maior grupo de transtornos do sono, com 24 distúrbios subdivididos em quatro subgrupos:

1. Distúrbios do acordar: envolvem alterações comportamentais associadas ao acordar, como o sonambulismo e o terror noturno.

2. Distúrbios da transição sono e vigília: envolvem alterações comportamentais durante o período de sono, como sobressaltos do sono e câimbras noturnas.

3. Parassonias associadas com o sono REM: envolvem alterações experienciadas exclusivamente durante o sono REM, como pesadelos e paralisia do sono.

4. Outras parassonias: incluem as demais alterações do sono, como bruxismo, síndrome da morte súbita noturna inexplicada e parassonias sem especificação.

\section{Distúrbios do sono relacionados a alterações médico-psiquiátricas}

Esses distúrbios reúnem 19 transtornos associados a doenças mentais ou neurológicas e outras afecções médicas:

1. Distúrbios associados a doenças mentais: ocorrem entre portadores de psicoses, transtornos de humor e transtornos da ansiedade, e podem alterar a qualidade e a continuidade do sono.

2. Distúrbios associados a enfermidades neurológicas: ocorrem entre portadores de doenças degenerativas cerebrais, demências, mal de Parkinson, insônia familiar fatal, epilepsia relacionada ao sono, estado de mal epilético de sono e cefaléia relacionada ao sono.

3. Distúrbios associados a afecções médicas: ocorrem entre portadores de alterações relacionadas aos sistemas cardiovascular, respiratório e digestivo (Reimão, 1996), como isquemia cardíaca noturna, doença pulmonar obstrutiva crônica, refluxo gastroesofágico relacionado ao sono e doença do sono.

\section{Distúrbios do sono propostos}

Distúrbios do sono propostos são aqueles que

522 englobam síndromes heterogêneas sem requisitos para definições específicas, como sono curto, sono longo, hiperidrose do sono e síndrome do engasgue no sono (Reimão, 1996; Reite, Ruddy \& Nagel, 2004).

\section{Prevalência}

A prevalência dos distúrbios do sono na população adulta é estimada em 15\% a 27\%, com cerca de 70 milhões de pessoas sofrendo algum tipo de distúrbio de sono só nos Estados Unidos. No Brasil, a estimativa de transtornos de sono na população geral é de aproximadamente dez a vinte milhões de pessoas (Martinez, 1999).

Os distúrbios de maior prevalência na população geral são a insônia e a síndrome da apnéia obstrutiva do sono (Roth et al., 2002). Na Coréia do Sul, Ohayon e Hong (2002) encontraram uma prevalência de insônia de um para cada cinco indivíduos na população geral e queixas de insatisfação com o sono em 10\% dos participantes, com maior prevalência entre mulheres e idosos. Zeitlhofer et al. (2000) encontraram resultados semelhantes com associação entre distúrbios do sono, sexo feminino e avanço da idade na população de cidades ocidentais industrializadas, onde a maioria dos distúrbios tinha características crônicas. Li, Wing e Fong (2002) também relataram uma incidência 60\% maior de insônia entre mulheres do que entre homens. É consenso entre os autores que a insônia compromete a qualidade de vida das pessoas (Janson, Lindberg, Gislason, Elmasry \& Boman, 2001; Littner et al., 2003; Smith \& Perlis, 2006).

No início desta década, Young, Peppard e Gottlieb (2002) associaram a apnéia ao maior consumo deálcool, tabagismo, congestão nasal, diminuição dos níveis de estrogênio e obesidade. Os autores enfatizaram que a literatura subestimava a prevalência desse transtorno e o número de casos não diagnosticados. Não obstante, a prevalência da apnéia obstrutiva do sono nos anos seguintes continuou estimada, de modo consistente, em 2\% das mulheres e 4\% dos homens (Culebras, 2006; Kryger, 2002; Neau et al., 2002; White, 2006).

Ao estudar a relação entre distúrbios respiratórios do sono, insônia crônica e menopausa entre mulheres não medicadas, Guilleminault, Palombini, Poyares e Chowdhuri (2002 a, b) encontraram que 83\% da amostra 
tinham distúrbios respiratórios do sono que provocavam o aumento da fadiga diurna. Dancey et al. (2003), estudando a relação entre distúrbios respiratórios e obesidade, encontraram maior freqüência e severidade de apnéia entre homens obesos do que entre mulheres. Em outro estudo, Young, Finn, Austin e Peterson (2003) concluíram que também entre as mulheres na maturidade, no período de pré, peri e pós-menopausa, a obesidade, avaliada pelo índice de massa corporal e circunferência do pescoço, é fator de risco para os distúrbios respiratórios. Esses autores concluem que o aumento do índice de massa corpórea e da circunferência do pescoço, associado à menopausa, aponta a idade e o peso como agravantes dos transtornos respiratórios. No estudo de Tribl et al. (2002), 75\% dos participantes acima de 50 anos, de ambos os sexos, apresentavam apnéia do sono e comorbidades com outros distúrbios, principalmente ronco, paradas respiratórias e movimento dos membros. Victor (2004) também relatou comorbidades entre portadores de apnéia, geralmente incluindo ronco, hipersonolência diurna e sobrepeso.

Ohayon e Roth (2002) investigaram a prevalência do transtorno de movimentos periódicos dos membros e da síndrome das pernas inquietas, conforme critérios da American Sleep Disorders Association (1997), em cinco países europeus. Os resultados mostraram prevalência de 3,9\% para os movimentos periódicos e de 5,5\% para a síndrome das pernas inquietas, além de forte associação desses distúrbios com a apnéia obstrutiva, cataplexia, e problemas clínicos como anemia, distúrbios afetivos, doenças cardiovasculares e problemas nas articulações. Saletu et al. (2001), em outro estudo, encontraram forte associação entre a síndrome das pernas inquietas e insônia, com prevalência de 5,0\% a 10,0\% na população geral e maior incidência entre mulheres e idosos.

O bruxismo é um transtorno caracterizado pela pressão ou ranger dos dentes, que ocorre pelo menos uma vez na vida de $85 \%$ a $90 \%$ da população, com prevalência estimada em 5\% a 8\% da população adulta (Bader \& Lavigne, 2000). Pessoas ansiosas e propensas ao estresse são consideradas mais vulneráveis ao bruxismo.

\section{Conseqüências dos distúrbios do sono}

A maioria dos distúrbios do sono não é detectada e tratada porque, em geral, as pessoas desconhecem que essa condição é clínica e tratável. Talvez em função desse desconhecimento, o paciente também deixa de relatar problemas de sono durante as consultas médicas, dificultando o acesso do profissional às informações que permitiriam o diagnóstico e o tratamento (Roth et al., 2002). Ohayon e Hong (2002) relataram que apenas 6,8\% de uma amostra estudada na Coréia do Sul buscaram assistência médica para tratar suas dificuldades com o sono.

As conseqüências dos distúrbios do sono envolvem questões econômicas e de saúde, como o aumento de hospitalizações, do absenteísmo, de riscos de acidentes de trânsito e de desenvolvimento de distúrbios mentais (Ohayon \& Smirne, 2002). Roberts, Roberts e Chen (2001, 2002) encontraram entre jovens com problemas de sono, comparados com seus pares com sono normal, maior incidência de depressão, ansiedade, irritabilidade, medo, raiva, tensão, instabilidade emocional, desatenção, problemas de conduta, uso de álcool e de outras drogas, ideação ou tentativa de suicídio, fadiga, falta de energia, dores de cabeça e de estômago e pior saúde. Em estudo semelhante, Roth et al. (2002) encontraram relatos de saúde precária, menos energia e pior funcionamento cognitivo entre portadores de distúrbios do sono quando comparados a pessoas com sono normal. Em geral, os estudos têm encontrado associação dos distúrbios do sono com problemas de saúde, funcionamento diário e bem-estar.

Distúrbios do sono estão associados também ao desencadeamento de transtornos psiquiátricos como depressão, freqüente entre portadores de insônia e de outros distúrbios do sono (Ancoli-Israel, 2006; Hublin, Kaprio, Partinen \& Koskenvuo, 2001; Morawetz, 2003; Rocha et al., 2002b; Walsh, 2004). Ohayon e Hong (2002) encontraram, entre pessoas com insônia, queixas de distúrbios respiratórios, de doenças cardíacas, insatisfação com a vida social, rebaixamento do funcionamento diário, doenças psiquiátricas, estilo de vida estressante e doenças físicas acompanhadas ou não de dor. Vicent e Walker (2000) verificaram que a preocupação excessiva com erros e precisão, excesso de ordem e organização, padrões e expectativas muito altos favoreciam o surgimento ou a piora da insônia, aumentavam a latência e reduziam o tempo do sono noturno. Os autores encontraram ainda maior preocupação com a possibilidade de erros e mais dúvidas sobre suas ações entre pessoas perfeccionistas insones. 
A insônia tem maior incidência entre pessoas separadas, do lar, desempregadas, ou aposentadas (Ohayon \& Roth, 2001), de baixa classe socioeconômica (Li et al., 2002) e costuma ser associada a doenças somáticas (Edinger, Wohlgemuth, Radtke, Marsh \& Quillian, 2001; Smith, Huang \& Manber, 2005), dor (Janson et al., 2001; Zammit, Weiner, Damato, Sillup \& McMillan, 1999) e distúrbios psiquiátricos (Babar et al., 2000; Hublin et al., 2001; Li et al., 2002). Esses dados ressaltam um segundo problema paralelo às perdas individuais, que é o aumento nos custos com serviços públicos de saúde devido aos transtornos do sono (Littner et al., 2003; Thase, 2005).

As perdas ocupacionais mais freqüentes, associadas à insônia, são o absenteísmo (Littner et al., 2003), a diminuição na qualidade (Zammit et al.,1999) e na produtividade do trabalho (Edinger et al., 2001; Leger, Massuel, Metlaine \& Sisyphe Study Group, 2006), o aumento de riscos de acidentes (Edinger et al., 2001) e a redução no número de promoções em relação a pessoas com sono normal (Hauri, 1998). Zammit et al. (1999) encontraram ainda, com menor freqüência, pouco otimismo em relação à carreira e empregos futuros, dificuldades para lidar com situações estressantes, tomar decisões, solucionar problemas e no relacionamento com colegas. Os déficits cognitivos provocados pelo distúrbio de sono como desatenção, baixa concentração e falhas de memória podem ainda agravar essas dificuldades (Buysse \& Ganguli, 2002; Day et al., 2001).

A insônia aparece associada também ao uso de substâncias como o álcool, nicotina (Janson et al., 2001), e medicações (Edinger et al., 2001; Li et al., 2002). O álcool, por ter propriedades sedativa e hipnótica, costuma ser utilizado para induzir o sono, mas causa fragmentação, alteração da arquitetura (Babar et al., 2000) e interrupção do sono (Harvey, 2002; Ohayon \& Hong, 2002), piorando sua qualidade (Eddy \& Walbroehl, 1999). Estudos com adultos jovens mostraram que 10\% (Eddy \& Walbroehl, 1999) a 30\% (Roehrs, Hollebeek, Drake \& Roth, 2002) dos participantes utilizavam medicações não prescritas e bebidas alcoólicas para dormir melhor.

Estudando pessoas com narcolepsia, Daniels, King, Smith e Shneerson (2001) verificaram que elas evitavam situações que pudessem envolver emoções, com receio de dormir em público ou de sofrer um ataque cataplético; apresentavam dificuldades para fazer

524 amigos; perda do emprego devido ao distúrbio; desempenho ruim no trabalho por falta de concentração e problemas de memória; dificuldades em realizar atividades diárias como cozinhar e cuidar de crianças e maior envolvimento em acidentes quando estavam dirigindo. Quase metade da amostra apresentava sintomas depressivos e relatava saúde inferior à da população geral, mesmo quando estavam em tratamento com anfetaminas ou drogas anticatapléticas.

A apnéia obstrutiva do sono é associada com ronco (Sundaram, Bridgman, Lim \& Lasserson, 2005), hipersonolência diurna (Chung, 2000; Bounhoure et al., 2005), hipertensão arterial (Alessi et al., 2002; Giles et al., 2006), cefaléias matinais ou em salvas (Nobre, Moreira \& Dominici, 2003), déficits cognitivos e enfermidades cardiovasculares (Martinez, 1999; Gus et al., 2002) e com alterações nos processos comportamentais, mentais e nas relações interpessoais (Brown, 2005). Portadores de apnéia costumam apresentar hipersonolência diurna resultante de microdespertares durante o sono, que aumenta a suscetibilidade a acidentes (Shamsuzzaman, Caples \& Somers, 2003) em até sete vezes quando se compara à propensão de pessoas com sono normal (Desai, Ellis, Wheatley \& Grunstein, 2003; McEvoy, 2003). Em crianças, provoca a diminuição do aprendizado e o aumento de distúrbios comportamentais (Mitchell, 2005). Ao despertar com a parada respiratória, o paciente pode ter dificuldade para retomar o sono, por temer um novo episódio (Edinger et al., 2001), o que aumenta a latência e a privação de sono, os cochilos diurnos (Hauri, 1998) e os riscos de acidentes diversos (Ayas et al., 2006; Cistulli \& Grunstein, 2005).

De acordo com Martinez (1999), a literatura mostra que $40 \%$ a $80 \%$ dos trabalhadores em turnos apresentam problemas para dormir. A principal causa desses problemas costuma ser a ruptura do ritmo circadiano, que é uma dessincronia entre o ciclo do sono e a temperatura corporal. Em conseqüência, esses trabalhadores dormem em média duas a quatro horas a menos do que trabalhadores diurnos e apresentam pior produtividade devido à sonolência excessiva durante o trabalho.

\section{Hipersonolência diurna}

A hipersonolência diurna, como demonstrado, é causada por múltiplos fatores e pode se tornar incapacitante (Gus et al., 2002; Young, 2004). Não obstante, médicos e pacientes costumam ignorar esse sintoma, 
o que dificulta o diagnóstico e deteriora a qualidade de vida do paciente acometido (Martinez, 1999).

Em situações de sonolência as pessoas desenvolvem estratégias para manter o estado de alerta, como receber ar frio no rosto e/ou lavá-lo com água fria (Hayashi, Masuda \& Hori, 2003), ingerir café, ouvir música alta e se expor à luz intensa. Essas estratégias, além de inúteis, são especialmente perigosas se utilizadas por motoristas, porque favorecem a perda do controle sobre o sono e a pessoa pode adormecer ao volante (Tufik, 2001). A exposição à luz intensa, comum entre trabaIhadores em turnos, bloqueia a secreção da melatonina que evita a diminuição da temperatura do corpo e mantém a pessoa alerta (Hayashi et al., 2003). A cafeína também diminui a secreção da melatonina (Shilo et al., 2002) e, se consumida em excesso ou em horários impróprios, aumenta o tempo de vigília e reduz o tempo do sono total (Souza \& Guimarães, 1999).

As conseqüências da hipersonolência diurna sobre a saúde incluem dores de cabeça, edema (Hublin et al., 2001), alterações nas funções imunológicas e endócrinas, como a elevação dos níveis de cortisol, responsável pelo agravamento de doenças como hipertensão e diabetes (Ferrara \& De Gennaro, 2001). Outras conseqüências incluem prejuízos na memória, na performance escolar, no humor e no comportamento; maior risco de doenças, acidentes, uso de drogas e desordens do sono em adolescentes (Liu \& Zhou, 2002); prejuízos cognitivos, afetivos (McCrae et al., 2003) e maior número de acidentes de trânsito entre idosos (Ohayon \&Smirne, 2002).

A hipersonolência diurna resulta também da privação intencional do sono. Desde o século passado, o homem reduziu em 20\% seu tempo total de sono para usar as horas de repouso na realização de outras atividades. Além de aumentar o número e os riscos de acidentes, a privação do sono ainda pode causar problemas cognitivos (Hublin et al., 2001), ocupacionais e motores, prejuízos nas relações familiares, sociais e pessoais (Reite, Ruddy \& Nagel, 2004) e alterações do humor (Buysse \& Ganguli, 2002).

A necessidade diária de sono depende das características e funcionamento diário de cada organismo, e da presença de déficits atribuídos ao número de horas de sono. Um indivíduo pode dormir oito horas por noite e mostrar indisposição, falhas de memória e hipersonolência diurna, enquanto outro pode dormir apenas cinco horas e mostrar total disposição e bem-estar (Ferrara \& De Gennaro, 2001; Martinez, 1999).

\section{Distúrbios do sono e qualidade de vida}

As conseqüências dos distúrbios do sono são fortemente relacionadas à qualidade de vida das pessoas que sofrem desse mal. O conceito de qualidade de vida proposto pela Organização Mundial de Saúde engloba domínios da saúde física, relações sociais, grau de independência, características ambientais, variáveis psicológicas e espirituais.

A qualidade do sono e a qualidade de vida estão intimamente relacionadas. O desemprego, por exemplo, é fator de qualidade de vida que pode afetar a qualidade do sono de um indivíduo porque a preocupação presente nessa situação aumenta a latência do sono e os despertares noturnos. Por outro lado, um indivíduo portador de distúrbio do sono provavelmente sofrerá conseqüências no trabalho devido à má qualidade do sono.

O desenvolvimento tecnológico contribui tanto de forma positiva quanto negativa para a qualidade de vida e a qualidade do sono das pessoas. Dentre os fatores que afetam o sono nas sociedades modernas estão a luz artificial - que modificou os hábitos relacionados aos ciclos sono-vigília e claro-escuro; a rede de telecomunicações, as demandas sociais e o estilo de vida ativo (Ferrara \& De Gennaro, 2001).

O sofrimento clínico da pessoa portadora de distúrbio de sono varia conforme os prejuízos ou conseqüências sofridas e a importância atribuída à área prejudicada. Com isso, a pessoa desenvolve estratégias para manejar essas dificuldades e melhorar suas condições e qualidade de vida. Os esforços para compensar os déficits percebidos remetem ao conceito de enfrentamento, que envolve a adaptação do organismo a situações adversas, como mudanças drásticas no curso da vida ou problemas que exigem novas formas de comportamento (Gimenes, 2000).

A nova demanda, em geral, provoca emoções, como ansiedade, culpa ou vergonha, que para serem removidas exigem respostas adaptativas. Para trabalhar em turnos alternados, por exemplo, uma pessoa necessita adaptar seus comportamentos ou ações a essa 
realidade. A mudança na rotina provoca alterações no sono e no funcionamento mental, físico, ocupacional e sexual do indivíduo que, em resposta, busca alternativas para restaurar o equilíbrio de seu sono e aliviar ou excluir os sintomas. Engle-Friedman et al. (2003) estudaram respostas à tarefa entre pessoas privadas de sono e concluíram que elas escolhiam tarefas menos difíceis e não acadêmicas e atividades matemáticas mais fáceis quando comparadas a pessoas não privadas de sono.

\section{Considerações Finais}

As conseqüências dos distúrbios de sono se desdobram em pelo menos três níveis subseqüentes que afetam a qualidade de vida da pessoa acometida. No primeiro nível estão as variáveis proximais ou biológicas, que trazem conseqüências imediatas ao organismo e incluem alterações fisiológicas como cansaço, fadiga, falhas de memória, dificuldade de atenção e de concentração, hipersensibilidade para sons e luz, taquicardia e alteração do humor.

No segundo nível estão as variáveis mediais ou funcionais, secundárias às conseqüências proximais, observadas a médio prazo. Elas têm implicações nas atividades cotidianas, como um primeiro desdobramento dos problemas com o sono, incluindo aumento do absenteísmo no trabalho, aumento de riscos de acidentes, problemas de relacionamento e cochilo ao volante.

No terceiro nível estão as variáveis distais ou extensivas, observadas a longo prazo, como um segundo desdobramento dos distúrbios do sono. Essas variáveis incluem a perda do emprego, seqüelas de acidentes, rompimento de relações, surgimento e agravamento de problemas de saúde.

Os estudos sobre distúrbios de sono e suas conseqüências mostram que as conseqüências em primeiro e segundo níveis são bastante semelhantes entre portadores dessa condição. São ainda necessários estudos detalhados sobre esses desdobramentos até o terceiro nível e o impacto deles na vida das pessoas de modo a oferecer uma real estimativa dos prejuízos pessoais e sociais conseqüentes aos distúrbios de sono. Com esses dados poderão ser planejados e elaborados programas específicos para prevenção e reabilitação da pessoa acometida, contemplando as diferentes

526 realidades.

\section{Referências}

Alessi, A., Alessi, C., Piana, E., Assis, M., Oliveira, L., \& Cunha, C. (2002). Influência da qualidade do sono na queda noturna da pressão arterial durante monitorização ambulatorial da pressão arterial. Arquivos Brasileiros de Cardiologia, 78 (2), 212-217.

American Psychiatric Association. (1994). Manual diagnóstico e estatístico dos transtornos mentais - DSM-IV-TR. Porto Alegre: Artes Médicas.

American Sleep Disorders Association. (1997). International classification ofsleep disorders, revised:diagnostic and coding manual. Rochester, Minn: American Sleep Disorders Association.

Ancoli-Israel, S. (2006). The impact and prevalence of chronic insomnia and other sleep disturbances associated with chronic illness. The American Journal of Managed Care, 12 (8), S221-S229.

Ayas, N. T., FitzGerald, J. M., Fleetham, J. A., White, D. P., Schulzer, M., Ryan, C. F., Ghaeli, R., Mercer, G., Cooper, P., Tan, M., \&Marra, C. (2006). Cost-effectiveness of continuous positive airway pressure therapy for moderate to severe obstructive sleep apnea/hypopnea. Archives of Internal Medicine, 166 (9), 977-984.

Babar, I., Enright, P., Boyle, P., Foley, D., Sharp, D., Petrovitch, H., \& Quan, S. (2000). Sleep disturbances and their correlates in elderly Japanese American men residing in Hawaii. Journal of Gerontology, 55 (7), 406-411.

Bader, G., \& Lavigne, G. (2000). Sleep bruxismo: an overview of an oromandibular sleep movement disorder. Sleep Medicine Reviews, 4 (1) 27-43.

Bounhoure, J. P., Galinier, M., Didier, A., \& Leophonte, P. (2005). Sleep apnea syndromes and cardiovascular disease. Bulletin de l'Académie Nationale de Médecine, 189 (3), 445-459.

Brown, W. D. (2005). The psychosocial aspects of obstructive sleep apnea. Seminars in Respiratory and Critical Care Medicine, 26 (1), 33-43.

Buysse, D., \& Ganguli, M. (2002). Can sleep be bad for you? Can insomnia be good? Archives of General Psychiatry, 59 (2), 137-138

Chung, K. F. (2000). Use of the Epworth Sleepiness Scale in Chinese patients with obstructive sleep apnea and normal hospital employees. Journal of Psychosomatic Research, 49 (5), 367-372.

Cistulli, P., \& Grunstein, R. R. (2005). Medical devices for the diagnosis and treatment of obstructive sleep apnea. Expert Review of Medical Advices, 2 (6), 749-763.

Culebras, A. (2006). Sindrome de apnea del sueno: soluciones a corto plazo y riesgo cerebrovascular a largo plazo. Revista de Neurología, 42 (1), 34-41.

Dancey, D. R., Hanly, P. J., Soong, C., Lee, B., Shepard, J., \& Hoffstein, V. (2003). Gender differences in sleep apnea. The role of neck circumference. Chest, 123 (5), 1544 -1550.

Daniels, E., King, M. A., Smith, I. E. \& Shneerson, J. M. (2001) Health-related quality of life in narcolepsy. Journal of Sleep Research, 10 (1), 75-81. 
Day, R., Guido, P., Helmus, T., Fortier, J., Roth, T., Koshorek, G., \& Rosenthal, L. (2001). Self -reported levels of sleepiness among subjects with insomnia. Sleep Medicine, 2 (2), 153-157.

Desai, A. V., Ellis, E., Wheatley, J. R., \& Grunstein, R. R. (2003). Fatal Distraction: a case series of fatal fall-asleep road accidents and their medicolegal outcomes. The Medical Journal of Australia, 178 (8), 396-399.

Eddy, M., Walbroehl, G.S. (1999). Insomnia. American Family Physician, 59 (7), 1911-1918.

Edinger, J. D., Wohlgemuth, W. K., Radtke, R. A., Marsh, G. R., \&Quillan, R. E. (2001). Does Cognitive-Behavioral insomnia therapy alter dysfunctional beliefs about sleep? Sleep, 24 (5), 591-599.

Engle-Friedman, M., Riela, S., Golan, R., Ventuneac, A., Davis, C., Jefferson, A., \& Major, D. (2003). The effect of sleep loss on next day effort. Journal ofSleep Research, 10, 75-81.

Ferrara, M., \& De Gennaro, L. (2001). How much sleep do we need? Sleep Medicine, 5(2), 155- 179.

Giles, T. L., Lasserson, T. J., Smith, B. H., White, J., Wright, J., \& Cates, C. J. (2006). Continuous positive airways pressure for obstructive sleep apnoea in adults. The Cochrane Database of Systematic Reviews, 3, Art: CD001106

Gimenes, M. G. (2000). A teoria do enfrentamento e suas implicações para sucessos e insucessos em psiconcologia. In M. G. Gimenes \& M. H. Fávero (Orgs.), A mulher e o câncer (pp.111-147). São Paulo: Livro Pleno.

Gottlieb, D. J., Yao, Q., Redline, S., Ali, T., \& Mahowald, M. W. (2000). Does snoring predict sleepiness independently of apnea and hypopnea frequency? American Journal of Respiratory and Critical Care Medicine, 162 (4), 1512-1517.

Guilleminaut, C., Palombini, L., Poyares, D., \& Chowdhuri, S. (2002a). Chronic insomnia, postmenopausal women and sleep disordered breathing. Part 1. Frequency of sleep disorders breathing in a cohort. Journal of Psychosomatic Research, 53 (1), 611-615.

Guilleminaut, C., Palombini, L., Poyares, D., \& Chowdhuri, S. (2002). Chronic insomnia, premenopausal women and sep disordered breathing. Part 2. Comparison of nondrug treatment trials in normal breathing and UARS post menopausal woman complaining of chronic insomnia. Journal of Psychosomatic Research, 53 (1), 617-623.

Gus, M., Nunes e Silva, D., Fernandes, J., Cunha, C., \& Sant'Anna, G. (2002). Epworth's sleepiness scale in outpatients with different values of arterial blood pressure. Arquivos Brasileiros de Cardiologia, 78 (1), 17-24.

Harvey, A. (2001). Insomnia: symptom or diagnosis? Clinical Psychology Review, 21 (7), 1037-1059.

Harvey, A. (2002). Identifying safety behaviors in insomnia. The Journal of Nervous and Mental Disease, 190( 1), 16-21.

Hauri, P. (1998). Sleep disorders: insomnia. Clinics in Chest Medicine, 19 (1), 157-168.

Hayashi, M., Masuda, A., \& Hori, T. (2003). The alerting effects of caffeine, bright light and face washing after a short daytime nap. Clinical Neurophysiology, 114 (12), 2268 -2278.

Hublin, C., Kaprio, J., Partinen, M., \& Koskenvuo, M. (2001). Insufficient sleep: a population-Based study in adults. Sleep: Journal of Sleep and Sleep Disorders Research, 24 (4), 392-400.
Janson, C., Lindberg, E., Gislason, T., Elmasry, A., \& Boman, G. (2001). Insomnia in men - a 10 year prospective population based study. Sleep: Journal of Sleep and Sleep Disorders Research, 24 (4), 425-430.

Kryger, M. (2002). What data do we need to diagnose and treat obstructive sleep apnea syndrome? Sleep Medicine Reviews, 6 (1), 3-6.

Leger, D., Massuel, M. A., Metlaine, A., \& SISYPHE Study Group (2006). Professional correlates of insomnia. Sleep, 29 (2), 171-178

Li, R., Wing, Y., \& Fong, S. (2002). Gender differences in insomnia: a study in the Hong Kong Chinese population. Journal of Psychosomatic Research, 53 (1), 601-609.

Littner, M., Hirshkowitz, M., Kramer, M., Kapen, S., Anderson, W., Bailey, D., Berry, R., Davilla, D., Johnson, S., Kushida, C., Loube, D., Wise, M., \& Woodson, T. (2003). Practice parameters for using polysomnography to evaluate insomnia: an update. Sleep, 26 (6), 754-760.

Liu, X., \& Zhou, H. (2002). Sleep duration, insomnia and behavioral problems among Chinese adolescents. Psychiatry Research, 111 (1), 75-85.

Martinez, D. (1999). Prática da medicina do sono. São Paulo: BYK.

Mc Evoy, R. D. (2003). Asleep at the wheel: who's at risk? The Medical Journal of Australia, 178 (8), 365-366.

McCrae, C. S., Wilson, N. M., Lichstein, K. L., Durrence, H. H., Taylor, D. J., Bush, A. J., \& Riedel, B. W. (2003). 'Young old' and 'old old' poor sleepers with and without insomnia complaints. Journal of Psychosomatic Research, 54 (1), 11-19.

McNicholas, W. T. (2003). Sleep apnoea syndrome today: much done, more to do. Sleep Medicine Reviews, 7 (1), 3-7.

Mitchell, R. B. (2005). Sleep-disordered breathing in children: are we underestimating the problem? European Respiratory Journal, 25 (2), 216 -217.

Morawetz, D. (2003). Insomnia and depression: which comes first? Sleep Research Online, 5 (2), 77-81.

Neau, J., Paquereau, J., Bailbe, M., Meurice, J., Ingrand, P., \& Gil, R. (2002). Relationship between sleep apnea syndrome, snoring and headaches. Cephalalgia, 22 (5), 333-339.

Nobre, M. E., Moreira, P., \& Dominici, M. (2003). Coexistência de cefaléias em salvas e apnéia do sono. Migrâneas Cefaléias, 6 (2), 46-52.

Novak, M., Shapiro, C. M., Mendelssohn, D., \& Mucsi, I. (2006). Diagnosis and management of insomnia in dialysis patients. Seminars in Dialysis, 19 (1), 25-31.

Ohayon, M. M., \& Hong, S. C. (2002). Prevalence of insomnia and associated factors in South Korea. Journal of Psychosomatic Research, 53 (1), 593-600.

Ohayon, M. M., \& Shapiro, C. M. (2002). Tenses of insomnia epidemiology. Journal of Psychosomatic Research, 53 (1), 525-527.

Ohayon, M. M., \& Smirne, S. (2002). Prevalence and consequences of insomnia disorders in the general population of Italy. Sleep Medicine, 3 (2), 115-120. 
Ohayon, M. M., \& Roth, T. (2001). What are the contributing factors for insomnia in the general population? Journal of Psychosomatic Research, 51 (6), 745-755.

Ohayon, M. M., \& Roth, T. (2002). Prevalence of restless legs syndrome and periodic limb movement disorder in the general population, Journal of Psychosomatic Research, 53 (1), 547-554.

Poyares, D., \& Tufik, S. (2002). I Consenso Brasileiro de Insônia. Hypnos Journal of Clinical and Experimental Sleep Research, 4 (2), 1-45.

Reimão, R. (1996). Sono: estudo abrangente (2a. ed.). São Paulo: Atheneu.

Reite, M., Ruddy, J., \& Nagel, K. (2004). Transtornos do sono (3a. ed.). Porto Alegre: Artes Médicas.

Roberts, R. E., Roberts, C. R., \& Chen, I. G. (2001). Functioning of adolescents with symptoms of disturbed sleep. Journal of Youth and Adolescence, 30 (1), 1-18.

Roberts, R. E., Roberts, C. R., \& Chen, I. G. (2002). Impact of insomnia on future functioning of adolescents. Journal of Psychosomatic Research, 53 (1), 561-569.

Rocha, F. L., Guerra, H. L., \& Lima-Costa, M. F. F. (2002). Prevalence of insomnia and associated sociodemographic factors in a brazilian community: the Bambuí study. Sleep Medicine, 3 (2), 121-126.

Rocha, F. L., Uchoa, E., Guerra, H. L., Firmo, J. O. A., Vidigal, P.G., \& Lima-Costa, M. F. (2002). Prevalence of sleep complaints and associated factors in community-dwelling older people in Brazil: the Bambuí Health and Ageing Study (BHAS). Sleep Medicine, 3 (3), 231-238.

Roehrs, T., Hollebeek, E., Drake, C., \& Roth, T. (2002). Substance use in Metropolitan Detroit. Journal of Psychosomatic Research, 53 (1), 571-576.

Roth, T., Zammit, G., Kushida, C., Doghramji, K., Mathias, S., Wong, J., \& Buysse, D. J. (2002). A new questionnaire to detect sleep disorders. Sleep Medicine, 3 (2), 99-108.

Saletu, M., Anderer, P., Saletu-Zyhlarz, G., Prause, W., Semler, B., Zoghlami, A., Gruber, G., Hauer, C., \& Saletu, B. (2001). Restless legs syndrome (RLS) and periodic limb movement disorder (PLMD): Acute placebo-controlled sleep laboratory studies with clonazepam. European Neuropsychopharmacology, 11 (1), 153-161.

Shamsuzzaman, A. S. M., Caples, S. M., \& Somers, V. K (2003). Sleep deprivation and circulatory control. Sleep, 26 (8), 934-935.

Shilo, L., Sabbah, H., Hadari, R., Kovatz, S., Weinberg, U., Dolev, S., Dagan, Y., \& Shenkman, L. (2002). The effects of coffee consumption on sleep and melatonin secretion. Sleep Medicine, 3 (3), 271-273.

Silber, M. H., Krahn, L. E., \& Olson, E. J. (2002). Diagnosing narcolepsy: validity and reliability of new diagnostic criteria. Sleep Medicine, 3 (2), 109-113.

Silverberg, D., Iaina, A., \& Oskenberg, A. (2002). Treating obstructive sleep apnea improves essential hypertension and quality of life. American Family Physician, 65 (2), 229-236.

Smith, M. T., \& Perlis, M. L. (2006). Who is a candidate for cognitive-behavioral therapy for insomnia? Health Psychology, 25 (1), 15-19.
Smith, M. T., Huang, M. I., \& Manber, R. (2005). Cognitive behavior therapy for chronic insomnia occurring within the context of medical and psychiatric disorders. Clinical Psychology Review, 25 (5), 559-592.

Souza, J. C., \& Guimarães, L. A. M. (1999). Insônia e qualidade de vida. Campo Grande: Editora UCDB.

Sundaram, S., Bridgman, S., Lim, J., \& Lasserson, T. J. (2005). Surgery for obstructive sleep apnoea. The Cochrane Database of Systematic Reviews, 4 Art: CD001004.

Thase, M. E. (2005). Correlates and consequences of chronic insomnia. General Hospital Psychiatry, 27 (2), 100-112.

Thorleifsdottir, B., Björnsson, J. K., Benediktsdottir, B., Gislason, Th., \& Kristbjarnarson, H. (2002). Sleep and sleep habits from childhood to young adulthood over a 10-year period. Journal of Psychosomatic Research, 53 (1), 529-537.

Tribl, G., Schmeiser-Rieder, A., Rosenberger, A., Saletu, B., Bolitschek, J., Kapfhammer, G., Katschnig, H., Holzinger, B., Popovic, R., Kunze, M., \& Zeitlhofer, J. (2002). Sleep habits in the Austrian population. Sleep Medicine, 3 (1), $21-28$

Tufik, S. (2001). O custo social do sono. Ciência Hoje, 170 (29), 6-10.

Victor, L. D. (2004). Treatment of obstructive sleep apnea in primary care. American Family Physician, 69 (3), 561-568.

Vincent, N. K., \& Walker, J. R. (2000). Perfectionism and chronic insomnia. Journal of Psychosomatic Research, 49 (5), 349-354.

Walsh, J. K. (2004). Clinical and socioeconomic correlates of insomnia. The Journal of Clinical Psychiatry, 65 (8), 13-19.

White, D. P (2006). Sleep apnea. Proceedings of the American Thoracic Society, 3 (1), 124-128.

World Health Organization (1997). Classificação de doenças mentais da CID-10 (10a. ed.). Porto Alegre: Artes Médicas.

Young, T. B. (2004). Epidemiology of daytime sleepiness: definitions, symptomatology, and prevalence. The Journal of Clinical Psychiatry, 65 (16), 12-16.

Young, T., Finn, L., Austin, D., \& Peterson, A. (2003). Menopausal status and sleep-disordered breathing in the Wiscosin Sleep Cohot Study. American Journal of Respiratory and Critical Care Medicine, 167 (9), 1181-1185.

Young, T., Peppard, P. E., \& Gottlieb, D. J. (2002). Epidemiology of obstructive sleep apnea: a population health perspective. American Journal of Respiratory and Critical Care Medicine, 165 (9), 1217-1239.

Zammit, G. K., Weiner, J., Damato, N., Sillup, G. P., \& McMillan, C. A. (1999). Quality of life in people with insomnia. Sleep: Journal of Sleep Research and Sleep Medicine, 22 (2), 379-385.

Zeitlhofer, J., Schmeiser-Rieder, A., Tribl, G., Rosenberger, A., Bolitschek, J., Kapfhammer, G., Saletu, B., Katschnig, H., Holzinger, B., Popovic, R., \& Kunze, M. (2000). Sleep and quality of life in the austrian population. Acta Neurologica Scandinavica, 102 (4), 249-257.

Recebido em: 25/5/2006

Versão final reapresentada em: 6/10/2006

Aprovado em: 24/11/2006 